\title{
Mesurer le temps en mer : instruments, voyages et échelles des mondes ibériques au XVI ${ }^{\mathrm{e}}$ siècle
}

The measurement of time at sea: instruments, voyages and scales in Iberian worlds in the $\mathrm{XVI}^{\text {th }}$ century

\section{Leonardo Ariel Carrió Cataldi}

\section{OpenEdition}

\section{Journals}

Édition électronique

URL : http://journals.openedition.org/artefact/453

DOI : $10.4000 /$ artefact.453

ISSN : 2606-9245

Éditeur :

Association Artefact. Techniques histoire et sciences humaines, Presses universitaires du Midi

Édition imprimée

Date de publication : 1 octobre 2016

Pagination : 235-247

ISBN : 978-2-7535-5174-9

ISSN : 2273-0753

Référence électronique

Leonardo Ariel Carrió Cataldi, « Mesurer le temps en mer : instruments, voyages et échelles des mondes ibériques au XVI e siècle », Artefact [En ligne], 4 | 2016, mis en ligne le 07 juillet 2017, consulté le 30 avril 2019. URL : http://journals.openedition.org/artefact/453 ; DOI : 10.4000/ artefact. 453 


\section{Mesurer le temps en mer: instruments, voyages et échelles des mondes ibériques au $x v I^{\mathrm{e}}$ siècle ${ }^{*}$}

Leonardo Ariel CARRIÓ CATALDI*

\section{Résumé}

À la croisée de l'histoire moderne des empires ibériques et de l'anthropologie des savoirs et des techniques, l'article propose une lecture sociale de l'emploi de différents instruments de mesure utilisés par D. João de Castro (1500-1548) lors de son voyage maritime de Lisbonne à Goa (1538) à bord du Grifo. Du bateau comme espace social au bateau comme " lieu de savoir », l'attention est portée sur l'utilisation d'un nouvel instrument, l'instrument des ombres, et sur la mesure du temps en mer.

Mots-clés : bateau, Castro (D. João de) (1500-1548), Empires ibériques, horloge universelle, instrument des ombres, lieu de savoir.

\section{Abstract. The measurement of time at sea : instruments, voyages and scales in Iberian worlds in the $\mathrm{XVI}^{\text {th }}$ century}

Drawing upon the history of Iberian modern empires, and the anthropology of knowledge and technics, the article proposes a social analysis of the employment of different measuring instruments by D. João de Castro (1500-1548) when sailing from Lisbon to Goa (1538) aboard the Grifo. From the ship as a social space to the ship as a space of knowledge, the paper focuses on the utilisation of a new device, the shadow instrument, and on the the measure of time at sea.

\footnotetext{
* Cet article a été rédigé à partir de certains des résultats et développements présentés dans un chapitre de ma thèse doctorale Temps, science et empire. Conceptions du temps au XVI e siècle dans les monarchies ibériques, SNS-EHESS, intitulé « Dom João de Castro ou le bateau comme lieu de savoir ». La perspective a été cependant modifiée.

**. Docteur en histoire de l'EHESS de Paris et de la SNS de Pise, Leonardo Ariel Carrió Cataldi a récemment soutenu sa thèse doctorale et est actuellement membre rattaché du Centre de recherches historiques (EHESS). Ses principaux axes de recherche sont l'histoire des sciences, des techniques et des instruments à l'époque moderne ainsi que l'historiographie contemporaine. Contact : [leonardo. carrio@ehess.fr].
} 
Keywords : Castro (D. João de) (1500-1548), Iberian empires, shadow instrument, ship, space of knowledge, universal clock.

\section{La " mystique du sang " ou le bateau comme espace social}

Mourir en mer était considéré, dans les sociétés chrétiennes d'Ancien Régime, comme une malemort-loin de la famille, parfois même d'un prêtre et sans avoir le temps de recevoir les derniers sacrements ou la possibilité de donner une sépulture, " topographie du souvenir", au défunt ${ }^{1}$. L'organisation sociale d'alors n'accordait pas la même importance à toutes les morts. Horizon pourtant commun à tous, une " mystique du sang ${ }^{2}$ " participait à l'instauration et à l'entretien d'un rapport asymétrique entre les différents groupes qui composent la société. Bonne ou mauvaise mort, en mer ou à terre, les élites sociales des royaumes se perpétuaient principalement par la « voix du sang $^{3}{ }^{\prime}$.

Dans le cas des monarchies ibériques et plus précisément de la couronne portugaise qui retiendra notre attention dans cet article, c'est notamment par la mer que, depuis le début du $\mathrm{xv}^{\mathrm{e}}$ siècle, le royaume s'étend hors du territoire péninsulaire ${ }^{4}$. Il déploie, grâce à l'ouverture de la route maritime reliant l'Europe et l'Asie (1497-1499, voyage de Vasco da Gama - c. 1460-1524), un « empire réseau ${ }^{5}$ ", l'Estado da Índia, entre le cap de Bonne-Espérance et le Japon. Presque en même temps, la couronne installe ses premières feitorias, enclaves visant à assurer l'échange commercial, au Brésil', reproduisant ainsi une modalité de présence similaire à celle des enclaves portugaises de la côte atlantique de l'Afrique. Entre 1497 et 1570, environ 613 navires auraient quitté le Portugal en direction principalement de villes-clés d'Inde telles que Goa et Cochin. Bien qu'au long des règnes de $\mathrm{D}$. Manuel I (1495-1521), de D. João III (1521-1557) et de D. Sebastião (1557-1578), le nombre de nefs baisse progressivement, leur envergure augmente. Chacun de ces bâtiments peut transporter entre 120 et 130 personnes ${ }^{7}$.

De la noblesse de sang aux marchands, en passant par les soldats, les marins et les différents membres du clergé, dans cet espace social flottant et en mouvement qu'est le bateau, la hiérarchie sociale qui structure et gouverne la société s'entrecroise avec celle basée sur une reconnaissance de la maîtrise des techniques nécessaires pour commander le navire. Une fois arrivés sur la terre ferme, ces groupes sociaux essaieront de trouver leur place au cœur des sociétés en transformation et qui modifient profondément, à leur tour, le microcosme social hétérogène existant à bord. Ce sera par le déploiement de cadres administratifs souvent précaires, par la négociation de leur rôle avec les autorités locales ou 
régionales, par le commerce, la confrontation armée, la cohabitation ou le mariage qu'ils essaieront de s'installer dans différentes régions $\mathrm{du}$ monde, d'exercer le pouvoir au nom d'un lointain roi et de répandre leur foi ${ }^{8}$. Mais, d'un port à l'autre, lors du voyage, le maintien et la reproduction des hiérarchies et des élites sociales, notamment de la noblesse de sang, dépendent de la bonne maîtrise de l'art de naviguer tout comme du hasard lié aux caprices, souvent imprévisibles, des vents et de la mer mortifère.

La « voix du sang » se perpétue, aussi en haute mer, par l'asymétrie. Les écarts que celle-ci établit et par lesquels elle survit émergent comme une réalité matérielle dès que l'on s'intéresse à ce que les différents membres de l'équipage peuvent embarquer pour conjurer la malemort. En 1509, le roi D. Manuel I (1469-1521), par un ensemble d'instructions, désigne le fonctionnement des « maisons » et " magasins » (Casas das Indias e Mina et leur armazéns), dont le but premier est le contrôle et le stockage des marchandises, où s'organisent le départ et l'arrivée des navires à Lisbonne'.

À titre d'exemple des inégalités dans les moyens pour affronter les difficultés du voyage, dans ces mêmes instructions, le roi réglemente également ce que les différents membres de l'équipage peuvent emporter pour faire le voyage jusqu'à São Jorge da Mina, enclave portugaise sur la côte africaine. En ce qui concerne la distribution des vivres essentiels tels que les " biscuits de mer » ou la viande, elle est inversement proportionnelle à l'effort physique que devront effectuer en mer les membres de l'équipage, du capitaine à l'apprenti marin. Seule la répartition du vin, des oignons et de l'ail est égalitaire. À chacun d'essayer de compenser les déficits d'apport nutritionnel par la pêche, le troc et le commerce pendant le voyage. La même réglementation accorde principalement au capitaine et au notaire le droit d'apporter à bord des vêtements de rechange et du linge pour se couvrir pendant la nuit. Cette distribution des vivres et des moyens pour se protéger des inclémences du climat en mer cherche à perpétuer la hiérarchie sociale. Depuis 1570, au moins officiellement, une ordonnance du roi D. Sebatião (1554-1578) stipule que le fournisseur responsable (provedor) de l'Armazém da Mina e Índia peut nommer les pilotes, les maîtres et les autres membres de l'équipage, mais le roi garde le droit de désigner le capitaine ${ }^{10}$. L'asymétrie sociale en haute mer peut ainsi garantir, d'un empire à l'autre, la circulation des élites ${ }^{11}$.

Quant à l'instruction des «gens de mer », les couronnes ibériques octroient différents titres liés à la pratique et à l'enseignement de la navigation et de la cosmographie. Ainsi, dans la documentation du début du xvI ${ }^{\mathrm{e}}$ siècle, on constate l'existence d'un " patron majeur », d'un " pilote majeur du royaume » et d'un " pilote majeur de la navigation de l'Inde et de la mer océan ${ }^{12}$ ». En 1547, Pedro Nunes (1502-1578) se voit attribuer le titre de " cosmographe majeur », comportant certaines responsabilités d'enseignement auprès des marins, peut-être déjà incluses dans les tâches à développer par le pilote majeur ${ }^{13}$. À partir des années 1560, il est certain que le cosmographe participe, avec un maître cartographe, à la validation des instruments, des cartes ainsi que des connaissances nautiques. Or, de manière générale, les fonctions des différents 
titres restent, cependant, peu précisées, notamment dans leur pratique. Les profils de ceux qui les obtiennent sont très hétérogènes, à l'image de la diversité des " gens de mer » que l'historiographie a peu abordés d'un point de vue social et professionnel, au-delà des noms les plus connus des «Grandes découvertes ${ }^{14}$ ».

C'est dans ce contexte historique d'expansion impériale de la couronne portugaise et d'organisation des savoirs nautiques, traversé par les logiques sociales et politiques décrites succinc- tement en amont, qu'il faut inscrire et comprendre le voyage maritime du noble portugais D. João de Castro de Lisbonne à Goa, en 1538, que nous analyserons dans cet article. Sous le prisme d'une perspective sociale de la littérature de voyage et de l'histoire des savoirs et des techniques et considérant le bateau comme un " lieu de savoir ", nous étudierons les enjeux de l'utilisation, lors de ce périple, de deux instruments, l'instrument des ombres, d'abord, et l'horloge universelle, ensuite ${ }^{15}$.

\section{Le bateau : espace social, "lieu de savoir »}

Le 6 avril 1538, part du port de Belém, à Lisbonne, une douzaine de nefs avec environ 2500 hommes en direction de $l^{\prime}$ Inde $^{16}$. Outre l'objectif diplomatique d'escorter le nouveau vice-roi et gouverneur de l'Estado da Índia (1538-1540), D. Garcia de Noronha (1479-1540), la flotte doit apporter son soutien militaire à l'enclave de Diu. Située dans une petite île de la région indienne du Gujarât, elle est stratégique pour le contrôle du commerce du golfe de Cambay et du golfe Persique. Les Portugais parviennent à $s^{\prime} y$ installer, après de nombreuses tentatives, grâce aux relations diplomatiques avec Bahâdur Shâh (?-1537), le sultan qui domine la région du Gujarât (15261537). En échange, ils lui proposent leur aide contre le deuxième empereur moghol de l'Inde, Humâyûn (1508-1556) qui a envahi le Gujarât (1535-1536), en obligeant le sultan à reculer jusqu'à Diu. Il semblerait que, parallèlement au dialogue avec les Portugais, Bahâdur Shâh ait fait appel au Grand Turc afin d'expulser ces derniers. Le grand amiral ottoman, Hadim Süleyman Pacha (ca. 1468-1548), à la tête d'une flotte, descend la mer Rouge vers $1^{\prime}$ Inde $^{17}$. Dans une situation complexe de tensions entre les différents pouvoirs présents dans la région, les Portugais s'emparent de la forteresse de Diu où ils résistent au siège de la flotte turque ${ }^{18}$. Celle-ci renonce finalement à l'offensive, le 5 novembre 1538, probablement à cause de rumeurs sur l'arrivée de la flotte de Noronha à Goa, le 11 septembre $1538^{19}$.

C'est à bord d'une de ces nefs, le Grifo, que D. João de Castro (15001548) embarque en tant que capitaine. Issu d'une famille noble liée à l'administration du royaume, beau-frère de Noronha, formé à l'art de la guerre et de la navigation dans les enclaves africaines de la monarchie portugaise et dans la lutte aux côtés de Charles Quint, contre Barberousse $^{20}$, Castro devient gouverneur (1545-1548) et vice-roi de l'Estado da Índia à la fin de sa vie (1548). 
Parmi les nombreux écrits et la documentation que Castro a laissés, nous retiendrons ici son premier roteiro, livre de bord où il retrace la route maritime suivie de Lisbonne à Goa en $1538^{21}$ et que Castro aurait, par ailleurs, retravaillé et complété lorsqu'il refait la même route en 1545. À bord de son bateau, le capitaine note minutieusement, jour après jour, les opérations des expériences menées pour se repérer en mer, la direction des vents ou la hauteur du soleil, ainsi que d'autres observations sur la météorologie et les peuples qu'il rencontre lors du périple. Cette structure temporelle scande et organise les informations d'un parcours qui nous amène $\mathrm{du}$ port portugais aux côtes indiennes. Le Grifo, espace social en mouvement, peut être ainsi analysé comme un « lieu de savoir $^{22}$ ». Autrement dit, un " système de relation ${ }^{23}$ » social au sein duquel s'opèrent des logiques de validation des techniques et des savoirs mobilisés par les différents acteurs dont Castro rend compte par l'écriture de son roteiro. Il est certain que le manuscrit se retrouve dans la péninsule avant la fin $\mathrm{du} \mathrm{XvI}^{\mathrm{e}}$ siècle. Nous ne connaissons que deux copies manuscrites incomplètes, alors conservées par les jésuites de la nouvelle université d'Évora, fondée en 1559. Une d'entre elles aurait appartenu au cardinal infant Henrique (1512-1580) ${ }^{24}$.

À Lisbonne, Castro correspond avec la cour de l'infant D. Luís (1506-1555) ${ }^{25}$. Frère du roi João III, D. Luís lui a offert, note Castro le 13 avril dans son roteiro ${ }^{26}$, l'instrument des ombres (estormento de sombras $)^{27}$, conçu par le cosmographe majeur du royaume, Pedro Nunes. Il en explique le fonctionnement dans un ouvrage réunissant plusieurs traités, publié un an avant le départ de Castro ${ }^{28}$. L'instrument sert à déterminer la latitude à des heures extra-méridiennes à partir de la hauteur du soleil et la variation de l'aiguille, c'est-à-dire l'écart entre le nord magnétique signalé par la boussole et le nord géographique. Ces deux données sont essentielles à la navigation hauturière. Alors que la première permet d'estimer la position nord-sud de la nef sans nécessairement dépendre de la hauteur du soleil à midi - comme c'est le cas lorsque l'on utilise la méthode traditionnelle ${ }^{29}$, la deuxième représente un problème majeur pour la navigation hauturière qu'il faut prendre en compte pour l'orientation correcte du navire. Dans ses rudiments les plus basiques, la navigation dite à l'estime repose fondamentalement sur la direction et la distance parcourue par le navire. Le portulan, composé d'une carte et d'un livre descriptif, peut venir à l'aide de ces deux paramètres d'orientation. Le calcul de la longitude, comme nous le verrons, reste un des grands problèmes de la navigation du XVI ${ }^{\mathrm{e}}$ siècle $\mathrm{e}^{30}$.

Cherchant à atteindre le cap de BonneEspérance, Castro prend note des opérations réalisées avec l'outil conçu par Nunes et d'autres instruments à l'appui, tel que l'astrolabe. En transférant les données obtenues sur une sphère graduée (poma), il parvient à obtenir la latitude à des heures extra-méridiennes. Suivant une procédure plus traditionnelle et en n'utilisant que l'astrolabe ou le quadrant, le reste de l'équipage ne peut quant à lui calculer la latitude qu'à partir de la hauteur du soleil à midi.

Sur le pont du navire, l'utilisation des instruments matérialise une scission sociale. D'un côté, D. João de Castro, 
noble et capitaine, ou capitaine parce que noble, emploie le quadrant et l'astrolabe, certes, mais reste vraisemblablement le seul à manipuler l'instrument des ombres. Conçu par le cosmographe royal et fabriqué par les mains d'un artisan de renommée européenne, l'instrument a été approuvé par l'infant $D$. Luis. C'est pour cette raison $\mathrm{qu}^{\prime}$ « il sera pertinent d'accorder foi à un instrument de si grande autorité, et ce que l'on trouve par lui doit être correct ${ }^{31} »$. De l'asymétrie sociale découle ainsi l'autorité d'un instrument de mesure auquel on doit croire car il s'agit, le mot n'a rien d'anodin, de foi.

De l'autre côté, entre les lignes du roteiro de Castro, on entrevoit le reste de l'équipage et les tensions au sein d'une entreprise qui est, à vrai dire, collective et plurielle. Tout au long du voyage, Castro et les membres de l'équipage comparent les résultats obtenus avec les différentes méthodes. Les tensions émergent principalement avec le pilote à bord, Manuel Álvares. Quelques jours après le départ, le 13 avril, Castro utilise l'instrument de Nunes. Le capitaine écrit les résultats obtenus le matin et dit les avoir donnés dans un « écrit fermé » (escrito çarrado), pour qu'il n'y ait pas de suspicions, au pilote qui réalisera les calculs le midi ${ }^{32}$. Magistrale façon de prétendre garantir les résultats face à un éventuel lecteur lointain, sur le pont du navire, le geste érige Castro comme celui qui dicte et organise la communication par laquelle les résultats s'établissent. Si, le 13 avril, la différence entre les résultats obtenus par le capitaine et le pilote sont très faibles, les écarts ne feront que s'accroître à mesure que le nombre de personnages qui déterminent la hauteur du soleil augmente. Castro hésite, pour un moment, sur la fiabilité de l'instrument, voire même sur la qualité des matériaux avec lesquels il a été fabriqué. En effet, le 17 juin, en considérant les différences entre les résultats obtenus, il critique notamment la poma. Pour Castro, les écarts sont dus à la rotondité inexacte de cette sphère graduée, au fait que son méridien, construit en laiton, soit mal gradué et que son « horizon», c'est-à-dire l'équateur, soit également peu précis. En général, écrit-il, « toutes ces choses " [les instruments] "sont fabriquées en toute hâte et sans prendre $\operatorname{soin}^{33} »$. Mais les différences sont finalement attribuées au pilote et aux horloges employées, comme nous le verrons.

\section{La difficile mesure du temps en mer et les échelles des mondes ibériques}

Le 17 avril 1538, Castro désespère des résultats obtenus. Il craint particulièrement que la « règle » pour trouver la hauteur du pôle en utilisant l'instrument de Nunes, expliquée dans le traité du cosmographe paru en 1537 , ne soit pas " générale », autrement dit, valable partout et tout le temps. Le 2 juin, Castro obtient en effet des résultats qu'il considère " impossibles » et "insupportables » (insoufrible) $)^{34}$. Il décide donc de mesurer la hauteur méridionale du soleil 
avec le reste de l'équipage. Sur le pont, un maître et deux marins se rassemblent et observent le soleil monter avec l'astrolabe jusqu'à ce que le pilote annonce que le soleil a atteint sa hauteur maximale. Sans trop de considérations et en lui faisant confiance, écrit Castro, tous prennent cette mesure comme référence et partent après avoir fini le relevé. Seuls Castro, le "docteur Luis Nunes », le pilote et le docteur, qu'il décrit comme un homme pratique et expérimenté, restent sur le pont. Ces derniers savent, explique le capitaine dans son roteiro, que c'est le pilote qui se trompe. Une heure plus tard, le soleil continue en effet à monter : les marins partis doivent refaire le calcul, le docteur interpelle le maître, le pilote doit donner raison à Castro.

"Les pilotes et hommes de mer", écrit Castro, ne prennent pas en compte le fait que les horloges - probablement des cadrans solaires dont l'angle du style doit être réglé en fonction de la latitude de l'endroit où il est utilisé - sont « faites en différentes régions et chacune sert à mesurer la hauteur du pôle de l'endroit où elle a été faite ${ }^{35}$ ». C'est la raison pour laquelle, poursuit-il, ils ne savent pas déterminer qu'il est vraiment midi et que le soleil atteint sa hauteur maximale. Ce midi-là, Castro dit avoir vérifié l'heure grâce à quatre horloges, dont deux marquaient des heures différentes. Par ailleurs, un autre facteur a une incidence sur les résultats. Les aiguilles ou boussoles que portent les horloges afin de les orienter selon les points cardinaux sont aimantées avec des pierres de différentes provenances, ce qui, selon Castro, associé à la composition du fer, produit des divergences dans la bonne indication du pôle magnétique. En effet, ce n'est que dans son voyage de Goa à Diu (1538-1539) que Castro pourra vérifier qu'une aiguille, qu'elle soit aimantée en Allemagne ou avec un aimant de la côte indienne, indique toujours le même nord. « Malgré le fait que les régions sont si différentes, la propriété de la pierre semble être la même ${ }^{36}$ ", écrira-t-il.

Castro est bien conscient du caractère local ou régional des outils de mesure. Un dernier exemple ne fait que conforter cette idée. Lors de son périple à Goa, toujours à la recherche impossible des coordonnées géographiques pour se repérer en mer, le 20 mai, le capitaine du Grifo prétend déterminer la longitude. Il veut le faire en suivant une procédure dont le principe est bien connu mais difficile à mettre en pratique, encore plus en haute mer. Il s'agit de mesurer le décalage horaire d'un phénomène, en l'occurrence d'une éclipse, observé en deux points distants et éloignés de la terre et de transformer la différence horaire en degrés de distance (une heure équivaut à 15 degrés). Pour ce faire, il a besoin d'une table avec le pronostic des éclipses ou d'un almanach, d'un calendrier pour confronter la date de la prédiction avec l'almanach et des horloges qui serviront à chronométrer l'heure de début et de fin de l'éclipse ainsi que sa durée ${ }^{37}$. Castro dit utiliser deux sabliers et un cadran solaire. Mais l'expérience échoue dès les préliminaires : après avoir mis, dans la mesure de ses possibilités, une table à niveau pour tenir le cadran solaire à l'horizontale, Castro confirme encore une fois qu'aucun des instruments ne mesure la même heure. Le capitaine indique que le problème réside dans le fait que les horloges qu'il utilise ont été fabriquées en Flandre et en Allemagne, et qu'il ne 
dispose pas $\mathrm{d}^{\prime}$ « horloge universelle » à ce moment-là ${ }^{38}$.

En effet, nous retrouvons une explication et une description de cette horloge universelle dans la littérature nautique des empires ibériques $\mathrm{du}_{\mathrm{xvI}}{ }^{\mathrm{e}}$ siècle, notamment dans un des best-sellers de l'époque, Breve compendio de la sphera y de la arte de navegar de Martín Cortés, publié à Séville en 1551. Il s'agit d'une horloge solaire placée sur un pied. Sur celui-ci est fixée une boussole - dont Castro avait indiqué le fonctionnement approximatif - permettant d'orienter l'horloge. Par un axe horizontal, appelé axe « du monde », il est possible d'ajuster son degré d'inclinaison par rapport au soleil et de mesurer ainsi la latitude sur un demi-cercle gradué placé verticalement derrière l'horloge ${ }^{39}$. C'est à travers ce type de technique de calibration que les utilisateurs d'instruments de mesure élaborés localement peuvent envisager l'idée que la mesure et l'instrument seront valables " ailleurs " et "partout $^{40} »$. Le choix de la variable qui servira d'étalon de mesure est un principe ou une "régulation sociale » à construire, " en ce qu'il constitue une convention partagée qui gouverne les mesures collectives et la manière dont elles offrent de nouvelles prises sur le monde ${ }^{41} »$. Il faut remarquer qu'il en va de même pour les échelles permettant de saisir ce monde et leurs multiples articulations en fonction des diverses trajectoires individuelles et sociales des acteurs qui les expérimentent dans des contextes historiques précis.
Fig. 1. - Martín Cortés, "Horloge Universelle», Breve compendio de la sphera..., Séville, 1551, fol. XLVII.1551, BnE. 


\section{Lire l'empire par la mesure}

Que ce soit dans le cas du projet impérial castillan ou portugais, les acteurs participant à la fabrique des savoirs cherchent à mesurer un monde qui semble se décomposer en différentes échelles dont les instruments portent les traces. Ou plutôt, le monde porte les traces des moyens que les acteurs se donnent pour le scruter.

Cortés l'exprime ainsi dans la dédicace de son traité à l'empereur Charles Quint :

« Et ainsi verront les vivants et comprendront ceux qui nous succéderont que le monde doit plus à Votre Majesté que l'Égypte à Isis. Elle leur a donné des lettres pour lire leurs cartes [" cartas»]. Votre Majesté leur donne des règles et la manière pour naviguer les mers. Le profit d'Isis était pour une seule province, l'utilité qui résulte d'ici est pour tout l'univers, pour toutes les provinces, pour toutes les mers, pour aller vers le découvert et pour découvrir ce qui est couvert ${ }^{42}$. »

Lire et naviguer par les langues et les instruments : les acteurs du $\mathrm{XvI}^{\mathrm{e}}$ siècle des couronnes espagnole et portugaise cherchent à rendre intelligible et mesurable un monde ouvert désormais aux quatre vents, pensé comme universel dans le cadre impérial et religieux des monarchies ibériques à l'époque moderne. Au caractère " provincial » de la langue comme technique pour explorer et se rapporter au monde, Cortés oppose les « règles " nautiques et la navigation comme étant d'une utilité universelle. Le geste d'arpentage sur lequel elles reposent permettrait un décloisonnement des échelles d'un empire de savoir qui se place entre ce qui est découvert et ce qui est encore à découvrir. Au sein de cette entreprise de mesure, présentée par opposition aux « lettres » données par Isis comme une sorte de langue universelle, la figure du traducteur, au sens large, semble perdre toute sa valeur face aux auteurs, qui, comme Cortés, établissent des manuels de l'art de naviguer et les instruments nécessaires.

À bord du Grifo, Castro se serait montré, très probablement, moins confiant dans les prétentions visées par Cortés, ou tout au moins il les nuancerait. Médiateur entre la cour et le bateau comme entre différentes techniques de mesure, c'est avant tout par l'écriture de son roteiro qu'il construit cette position d'intermédiaire. Dans quel but, finalement, écrire et décrire le monde depuis la mer, si ce n'est pas pour garder les traces de ce que les vagues vont aussitôt effacer?

Point de repère au milieu de l'immensité de l'océan, en écrivant les expériences réalisées au sein de ce microcosme social en mouvement, Castro fait $\mathrm{du}$ bateau un " lieu de savoir " dont l'existence survivra à la fin du voyage et au-delà du bastingage du Grifo grâce à son roteiro. Pour les lecteurs de ce texte, le Grifo devient ainsi observable, lisible. Comme Adir Ophir et Steven Shapin l'ont souligné pour le xvII siècle, l'insistance sur l'observation et l'expérimentation est une fonction des " espaces de savoir » : commençant à se " ségréguer » de l'ordinaire, on y force l'invisible à se manifester. Le Grifo et Castro, chargé 
d'expérimenter avec l'instrument des ombres, un instrument investi d'une très grande autorité sociale, peuvent être saisis sous cet angle d'analyse.

Comme le capitaine l'écrit en introduction de son roteiro, ce sont les muses, protectrices des savoirs, mais aussi celles qui révèlent aux yeux $\mathrm{du}$ poète le mystère pour pouvoir voir au-delà du présent ${ }^{43}$, qui devront aider les cosmographes à comprendre son roteiro. De même, la déviation de l'aiguille est un "secret " que le pilote souhaite comprendre ${ }^{44}$ et les résultats des mesures circulent entre les membres de l'équipage du Grifo dans des « écrits fermés ». L'invisible « apparaît seulement aux yeux de ceux qui sont autorisés à l'observer ${ }^{45}$ ». Participant actif de la constitution et de l'entretien de l'asymétrie sociale qui structure les sociétés d'Ancien Régime, Castro est le maître de cette " cérémonie de la mesure $^{46}$ » en haute mer et l'auteur du scénario au sein duquel elle trouve son sens premier.

\section{Notes}

1. Alain Cabantous, Le Ciel dans la mer. Christianisme et civilisation maritime, $\mathrm{XVI}^{e}$-XIX ${ }^{e}$ siècle, Paris, Fayard, 1990, p. 114; Romain BerTRAND, "Puisque ton navire n'est pas fait de planches... Métrologies nautiques et conjuration du péril spirituel en situation de "premiers contacts" (HollandeInsulinde, $\mathrm{XVI}^{\mathrm{e}} \mathrm{xVII}^{\mathrm{e}}$ siècle) ", Ethnologie française, 1, vol. 45, 2015, p. 19-29. Sur la vie quotidienne à bord d'un bateau au $\mathrm{XvI}^{\mathrm{e}}$ siècle, voir également Pablo Emilio Pérez-Mallaína Bueno, Los hombres del oceano. Vida cotidiana de los tripulantes de las flotas de Indias. Siglo XVI, Séville, Sociedad estatal para la Exposición universal Sevilla 92, Diputación de Sevilla, 1992; Francisco Contente Domingues et Inácio Guerreiro, " A vida a bordo na carreira da Índia (século xvI) », Separata da Revista da Universidade de Coimbra, vol. 34, 1988, p. 185-225; idem, "Viver a Bordo », Oceanos, 2, 1989, p. 37-63; Inácio GUerReIro, "Particularidades da vida no mar », Revista Oceanos, 38, 1999, p. 149-157.
2. Lucien Febvre, "La voix du sang. Fin d'une mystique? ", Annales. ESC, 2, vol. 4, 1949, p. 149-151.

3. Ibid.

4. L'historiographie s'accorde à considérer la date de la conquête de la ville de Ceuta, en 1415, comme le début de l'expansion maritime du royaume de Portugal.

5. 1505, date de désignation du premier vice-roi (visorey das Índias), D. Francisco de Almeida, est considérée comme le moment constitutif de l'Estado da Índia en tant qu'entité politique. Cette entité est définie, par Luís Filipe Thomaz, comme un réseau plutôt que comme un espace. L.F. ThомAZ, " Estrutura politica e administrativa do Estado da Índia no século xvI ", dans id., De ceuta a Timor, Lisbonne, Difel, 1994 [1985], p. 517; id., "L'idée impériale manuéline ", dans Jean Aubin (éd.), La découverte, le Portugal et l'Europe : actes du colloque, Paris, Centre Culturel Portugais, Fondation Calouste Gulbenkian, 1990, p. 35-103.

6. Pour un travail de synthèse sur l'expansion portugaise, voir, par exemple, Francisco Bethencourt et Kirti Chaudhuri (dir.), História da expansão portuguesa, 5 vol., Lisbonne, Círculo de leitores, 1998. Pour des approches plus spécifiques, mais toujours dans une perspective de synthèse, consulter Charles Boxen, The Portuguese Seaborne Empire, 1415-1825, Londres, Hutchinson, 1969; Sanjay Subrahmanyam, The Portuguese Empire in Asia, 1500-1700, 2e éd., Chichester, Wiley-Blackwell, 2012 [1993]; Donald F. LACH, Asia in the Making of Europe, v. 1, Chicago/Londres, University of Chicago Press, 1965; Michael Naylon Pearson, The New Cambridge History of India, vol. 1, The Portuguese in India, Cambridge/New York, Cambridge University Press, 1987.

7. F. Bethencourt et K. Chaudhuri (dir.), op. cit., vol. 1, A formação do Império (1415-1570), Lisbonne, Círculo de leitores, 1998, p. 229.

8. Pour des glossaires non exhaustifs des différents groupes sociaux en circulation à l'intérieur de l'empire portugais, voir S. SUBRAHMANYAM, op. cit., p. 230-231 et C. Boxer, op. cit., "Glossary ». Pour une approche sociale de l'Estado da Índia, voir les nombreux travaux de Dejanirah Couto, entre autres, "Quelques observations, sur les renégats portugais en Asie au Xvi ${ }^{e}$ siècle ", Mare Liberum, 16, 1998, p. 57-85.

9. L'ensemble d'instructions, dont la chronologie s'étale au-delà du royaume de D. Manuel I, est connu sous le nom de Regimento das cazas. Cette documentation a été publiée par Damião PERES, Regimento das cazas das Índias e Mina, Coimbra, Faculdade de Letras da Universidade de Coimbra, 1947. Pour une étude plus détaillée des différents « lieux de savoirs » nautiques et cosmographiques 
dans la péninsule Ibérique au $\mathrm{XvI}^{\mathrm{e}}$ siècle, je me permets de renvoyer ici à ma thèse de doctorat, op. cit., et à la bibliographié citée dans ce travail.

10. Ce document est cité par Joel SERRÃo, «India, armazém de ", Dicionário de história de Portugal, vol. III, Lisbonne, Iniciativas editorais, 1965, p. 504505.

11. Sur la circulation des élites comme mécanisme d'articulation des réseaux impériaux hispaniques et quelques exemples du monde portugais, voir Bartolomé Yun-CasaliLla, La redes del imperio : élites sociales en la articulación de la Monarquía Hispánica, 14921714, Madrid, Pons, Séville, Universidad Pablo de Olavide, 2009. Pour un aperçu synthétique du monde asiatique, voir S. SUBRAHMANYAM, op. cit., p. 22-26.

12. Avelino Teixeira da Mota, "Os regimentos do cosmógrafo-mor de 1559 e 1592 e as origens do ensino náutico em Portugal ", Junta de Investigações do Ultramar, 1969, p. 5-69.

13. Ibid., p. 8.

14. Voir notamment Amélia Polónia da Silva, "Os náuticos das carreiras ultramarinas ", Oceanos, 38,1999 , p. 113-128, et "L'historiographie portugaise sur les gens de mer et les communautés littorales. Une approche de synthèse ", dans Revue d'histoire maritime, 10-11, 2010, ainsi que la littérature à laquelle elle renvoie.

15. Les liens entre instruments de mesure et littérature de voyage ont été explorés, pour une époque postérieure à celle abordée ici, par Marie-Noëlle BOURGUET et Christian Licoppe : « Voyages, mesures et instruments. Une nouvelle expérience du monde au Siècle des lumières ", Annales HSS, 52-5, 1997, p. 1115-1151, et Marie-Noëlle Bourguet, Christian Licoppe et H. Otto Sibum (éd.), Instruments, travel and science : itineraries of precision from the seventeenth to the twentieth century, Londres/New York, Routledge, 2002.

16. José Manuel GArcia, « D. João de Castro : um homem de guerra e ciência ", dans Francisco Faria Paulino (coord.), Tapeçarias de D. João de Castro, [Lisbonne], Comissão Nacional para as Comemorações dos Descobrimentos Portugueses, Instituto Português de Museus, 1995, p. 15; Sylvie Deswarte-Rosa, «Espoirs et désespoir de l'infant D. Luís ", Mare Liberum, 3, Lisbonne, 1991, p. 241298 , suggère que deux mille hommes ont participé à cette entreprise.

17. Voir Stephan Michaelsen, "The Ottoman Expedition to Diu in 1538 ", Mare liberum, 9, 1995, p. 209-215.

18. La situation géostratégique se complexifie lorsque Humâyûn doit replier ses forces à cause de la pression militaire de Sher Shâh Sûri, qui est en train de regrouper les Afghâns dans le Bihâr, au nord-est du continent. Voir sur ce passage Louis FrÉDÉRIC,
Akbar, le Grand Moghol, Paris, Denoël, 1986, p. 22. Par ailleurs, en 1537, le sultan Bahâdur Shâh meurt dans des circonstances obscures alors qu'il débarquait d'un bateau portugais. Voir F. BETHENCOURT et K. Chaudhuri (dir.), op. cit., p. 179.

19. C'est J.-B. Aquarone, dans D. João de Castro, gouverneur et vice-roi des Indes orientales, 1500-1548..., t. 2, Montpellier, Déhan, 1967, p. 163, qui propose cette hypothèse. Voir également S. Michaelsen, art. cité, p. 214, et Dejanirah Couto, «Em torno da concessão e da fortaleza de Baçam (1529-1546) ", Mare liberum, 9, 1995, p. 117-132.

20. On sait que Castro est nommé chevalier à Tanger (où il aurait habité entre 1518-1527), et qu'il est capitaine d'un navire qui amène des hommes, vivres et munitions à Safi, en 1534. L. Albuquerque, Navegadores, viajantes e aventureiros portugueses, vol. II, Lisbonne, Caminho, 1987, p. 106; voir J.-B. Aquarone, op. cit., p. 36-42 sur Tanger et p. 53-54 sur Safi. Selon ce dernier, le séjour à Tanger a lieu entre 1518 et 1524 . Castro participe en 1535 comme capitaine d'une des 35 nefs avec lesquelles João III de Portugal prétend aider Charles Quint à récupérer la Tunisie, royaume de la dynastie berbère des Hafsides tombé entre les mains de Barberousse à peine un an auparavant. Les pages écrites par Fernand Braudel sur ce sujet sont encore d'une grande utilité. F. BRAUdel, La Méditerranée et le monde méditerranéen à l'époque de Philippe II, t. 3, $9^{e}$ éd., Paris, Armand Colin, 1990 [1949].

21. Nous utiliserons ici l'édition de João DE Andrade Corvo (éd.), Roteiro de Lisboa a Goa por D. João de Castro, Lisbonne, Academia Real das Sciencias de Lisboa, 1882. Il est toujours intéressant de consulter l'édition d'Armando CoRTESÃo et Luís De Albuquerque (éd.), Obras completas de D. João, 4 vol., Coimbra, Academia Internacional da Cultura Portuguesa, 1968-1971, où sont réunis les autres roteiros de Castro : De Goa a Diu (1539) et Do mar Roxo (1540-1541). Ces textes nautiques méritaient d'être analysés et contextualisés dans l'ensemble de roteiros publiés par Abel Fontoura da Costa, Roteiros portugueses inéditos da carreira da Índia do século XVI, Lisbonne, Agência Geral das Cólonias, 1940, notamment par rapport au Roteiro para a Índia e oriente, anonyme (1530, ca.) et les roteiros de Manuel Álvares (avec celui de Diogo Afonso, 1545 ca.). Nous remercions Dejanirah Couto d'avoir attiré notre attention sur cette référence. Sur la correspondance de D. João de Castro, Elaine SANCEAU (éd.), Cartas de D. João de Castro, Lisbonne, Agência Geral do Ultramar, 1954, et Luís DE AlbuQUeRQue (éd.), Cartas trocadas entre D. João de Castro e os filhos (1546-1548), Lisbonne, Comissão Nacional para as Comemorações dos Descobrimentos Portugueses, Ministério da Educação, 1989. Pour une discus- 
sion sur les roteiros en tant que "genre ", Avelino TeixeIrA DA MotA, «Evolução dos roteiros portugueses durante o século XVI ", Revista da Universidade de Coimbra, vol. XXIV, 1969; Abel FontourA DA CostA, « Bibliografia náutica portuguesa até 1700 » et « Os roteiros portugueses até 1700 » dans A Marinharia dos Descobrimentos, Lisbonne, Arenada, 1933; Joaquim Barradas de Carvalho, $\grave{A}$ la recherche de la spécificité de la Renaissance portugaise..., Paris, Fondation Calouste Gulbenkian, 1983; João Rocha Pinto, «A viagem, memória e espaço. A literatura portuguesa de viagens. Os primitivos relatos de viagem ao Índico 1497-1550 », Revista de história económica e social, 11-12, 1989; et, du même auteur, "Houve diários de bordo durante os séculos xv e xvi? ", Revista da Universidade de Coimbra, 34, 1987, p. 383-416.

22. L'expression « lieux de savoir » renvoie, dans le panorama historiographique français, à l'entreprise collective dirigée par Christian ЈАсов : Lieux de savoir, 2 vol., Paris, Albin Michel, 2007-2010. L'auteur a précisé les enjeux méthodologiques de son approche et d'une anthropologie historique des savoirs dans Qu'est-ce qu'un lieu de savoir?, Marseille, Open Édition Press, 2014. Pour une perspective plus large sur l'importance d'une approche spatiale en histoire des sciences voir, entre autres, Adi OpHIR et Steven SHAPIN, "The Place of Knowledge : a Methodological Survey ", Science in Context, 4 (1) 1991, p. 3-21; Jean-Marc BESSE, « Le lieu en histoire des sciences. Hypothèses pour une approche spatiale du savoir géographique au $\mathrm{XvI}^{\mathrm{e}}$ siècle ", Mélanges de l'École française de Rome - Italie et Méditerranée, Rome, École française de Rome, 116 (2), 2004, p. 401-422; id., «Approches spatiales dans l'histoire des sciences et des arts ", L'Espace géographique, 39 (3), 2010, p. 211224; José PARdo TOMÁs, Un lugar para la ciencia : escenarios de práctica científica en la sociedad hispana del siglo XVI, La Orotava, Fundación canaria orofava de historia de las ciencias, 2006. Les possibilités analytiques de l'articulation, dans un "jeu d'échelles », de « lieux » de savoirs comme approche pour comprendre la production et circulation de ces derniers, ont été mises à l'œuvre récemment dans Antonella Romano et Sabina Brevaglieri (dir.), " Produzione di saperi. Costruzione di spazi », dossier thématique de Quaderni storici, 142 (1), 2013. Pour la distinction entre «lieu » et « espace » comme «lieu pratiqué », se reporter à Michel DE CERTEAU, L'invention du quotidien. 1/Arts de faire, Paris, Gallimard, 1990 [1980], p. 172-175. Pour une perspective plus générale et de synthèse sur le "spatial turn ", voir Barney WARF et Santa ArIAs (éd.), The Spatial Turn. Interdisciplinary perspectives, Londres, Routledge, 2009.

23. С. ЈАсов, Lieux de savoir, op. cit., p. 69.
24. Il s'agit des manuscrits $C V X / 1-24$ et CVX/1-25 de la Bibliotheca publica Eborense. Le premier est composé de 106 feuilles $(29 \times 20,5 \mathrm{~cm})$ et contient également neuf dessins dont sept sont des représentations topographiques et les deux autres des phénomènes atmosphériques.

25. Sur l'infant D. Luís, voir Sylvie DeswarteRosA, art. cité, et la bibliographie que l'auteur cite en p. 244-245.

26. D. JoÃo de CAstro, op. cit., p. 28-30.

27. Pour une description du point de vue technique de cet instrument, voir L. DE AlbuQuerQue, Estudos de história, vol. IV, Coimbra, Acta Universitatis Conimbrigensis, 1976. Pour plus d'information sur ce sujet, consulter Luciano PEREIRA DA SILVA, «A arte de navegar dos portugueses desde o Infante a D. João de Castro », dans Facultade de Ciências da Universidade de Coimbra (éd.), Obras completas de Luciano Pereira da Silva, vol. 2, Lisbonne, Agência Geral das Colónias, Lisbonne, 1943-1946, p. 224-432; António EstÁcio Dos ReIs, « Os instrumentos de medida de Pedro Nunes ", 49, Oceanos, 2002, p. 20-34 et, dans la même perspective internaliste, Jean Widemann, Le nonius de Pedro Nunes et la mesure de la latitude au Portugal du XvI siècle, Lille, Atelier national de reproduction des thèses, 2006. Pour une révision plus récente qui corrige certains passages de l'étude de L. de Albuquerque, voir Paulo Jorge Antunes Nunes, Os instrumentos náuticos na obra de Pedro Nunes, mémoire de master en histoire maritime, Lisbonne, Universidade de Lisboa, Lisbonne, 2012.

28. Pedro Nunes, Tratado da sphera com a Theorica do sol e da lua..., Lisbonne, Germão Galharde, 1537. Édition moderne : P. NunEs, Obras, vol. 1, Lisbonne, Fundação Calouste Gulbenkian, 2002. La publication contient plusieurs traités. Les questions liées à l'utilisation de l'instrument sont développées notamment dans Tratado em defensam da carta de marear.

29. Sur les différentes méthodes pour calculer la latitude en mer, voir António Estácio Dos REIs, Medir estrelas. Measuring stars, Lisbonne, CTT Correios, 1997.

30. Sur la navigation au $\mathrm{XVI}^{\mathrm{e}}$ siècle, voir, par exemple, Abel Fontoura da Costa, A marinharia dos descobrimentos, Lisbonne, Imprensa da Armada, 1933. Sur la question de la longitude, l'historiographie reste encore centrée sur le problème technique et apporte, souvent, une perspective principalement évolutive. Entre autres travaux, William GRAHAM LISTER RANDLES, «Portuguese and Spanish Attempts to Measure Longitude in the $16^{\text {th }}$ Century ", Separata do Boletim da Biblioteca da Universidade de Coimbra, 39, 1984, p. 143-160 ; António Estácio dos REIs, « O problema da determinação da longitude no tratado de 
Tordesilhas », Mare Liberum, 8, 1994, p. 19-32; Jullien Vincent (dir.), Le calcul des longitudes. un enjeu pour les mathématiques, l'astronomie, la mesure du temps et la navigation, Rennes, PUR, 2002.

31. D. JoÃo de CASTRO, op. cit., p. 229.

32. Ibid, p. 35-38.

33. Ibid., p. 210.

34. Ibid., p. 181.

35. Ibid., p. 182-183.

36. Cité par A. Corvo, ibid., p. 184-185, note 1.

37. Les différences entre ces types d'imprimés sont parfois, au $\mathrm{XVI}^{\mathrm{e}}$ siècle, peu claires. Il s'agit le plus souvent de sources composites. Mais, de manière générale, on peut dire que les calendriers visent l'organisation de l'année ecclésiastique. Les almanachs précisent la position des planètes et organisent le rythme des activités quotidiennes. Les pronostics, souvent annexes aux almanachs, prévoient des phénomènes atmosphériques ou de salubrité, notamment à caractère catastrophique. Sur cette littérature, voir, par exemple, Biblioteca Nacional, Os sucessores de Zacuto. O almanaque na Biblioteca Nacional do século XV ao XXI, Coordenação Rosa Maria GALVÃo, apresentação João Luís LisboA, Lisbonne, Biblioteca Nacional, 2002.

38. Ibid., p. 147-153.

39. Nous n'avons pas pu vérifier le fonctionnement de cet instrument et, à notre connaissance, aucune étude ne l'a jusqu'à présent analysé de manière exhaustive. C'est l'angle formé par le style et le cadran qui devrait pouvoir se régler, mais Cortés semble suggérer que c'est l'inclination du cadran qui est réglée en fonction de la latitude.
40. Nous reprenons ici l'argument de Simon Schaffer, «Les cérémonies de la mesure. Repenser l'histoire mondiale des sciences ", Annales HSS, 2, avril-juin 2015, p. 409-435.

41. Ibid.

42. " $Y$ asi vera[n] los vivos y ente[n]dera[n] los q[ue] nos succediere[n] q[uan] to mas d[e]ve el mu[n]do a V.M. q[ue] Egipto a su ysis : ella les dio letras p[ar]a leer sus cartas $V$. M. les da reglas y manera p[ar]a navegar los mares. El provecho de isis fue para sola vna p[ro] vincia, la utilidad que d[e] aq[ui] resulta es p[ar]a todas provincias $p$ [ar]a todos mares p[ar]a yr a lo d[e]cubierto y p [ar] a d[e]cubri lo encubierto ", Martín ConTÉs, Breve compendio de la sphera y de la arte de navegar, con nuevos instrumentos y reglas, exemplificado con muy subtiles demostraciones, Séville, Antonio Álvarez, 1551, fol. IIIv ${ }^{\circ}$, BnE.

43. Jean-Pierre Vernant, Mythe et pensée chez les Grecs. Études de psychologie historique, Paris, La Découverte, 1996 [1965], p. 111-117.

44. D. JoÃo de CASTRo, op. cit., p. 296.

45. "Seventeenth-century emphasis on observation and experimentation may mask a major function of these segregated places: forcing the invisible to manifest itself, to leave traces, to betray a hidden presence. Yet the invisible appears only to the eyes of those authorized to observe it. The heterotopic site is at one and the same time a mechanism of social exclusion and a means of epistemically constituting conditions of visibility. » A. OpHIR et S. SHAPIN, art. cité, p. 13-14.

46. S. SCHAFFER, art. cité. 